\title{
Coil embolisation of a septal artery in a patient with hypertrophic obstructive cardiomyopathy
}

Yee Sen Chan Wah $\mathrm{Hak}^{1}$, Charles $\underline{\mathrm{Chan}}^{2}$, FRCP, FACC

\begin{abstract}
Nonsurgical septal reduction by coil embolisation of the first major septal branch was successfully performed in a 34-year-old patient with hypertrophic obstructive cardiomyopathy, resulting in acute reduction of left ventricular outflow tract obstruction and symptomatic improvement. Follow-up at 18 months showed sustained clinical and echocardiographic benefits from the procedure. To the best of our knowledge, this is the first case reported in Singapore.
\end{abstract}

Keywords: coil embolisation, hypertrophic obstructive cardiomyopathy, nonsurgical septal reduction

\section{INTRODUCTION}

Surgical myectomy is an established treatment in symptomatic patients with hypertrophic obstructive cardiomyopathy (HOCM) refractory to medical treatment. ${ }^{(1,2)}$ In nonsurgical patients, alcohol septal ablation is used as an alternative to surgical myectomy, and has been shown to effectively reduce left ventricular outflow tract (LVOT) obstruction and relieve symptoms. However, this treatment is limited by a high incidence of complete heart block, which requires permanent pacing. More recently, septal reduction by coil embolisation in order to avoid alcohol toxicity has been proposed as an alternative.

\section{CASE REPORT}

A 34-year-old Chinese male patient was referred to our hospital with a history of presyncope and increasing dyspnoea on exertion. He was diagnosed with HOCM three years ago at another institution and treated with atenolol $25 \mathrm{mg}$ twice daily (bd) and a prophylactic implantation of an automatic internal cardiac defibrillator. There was no family history of HOCM or sudden death. On physical examination, a grade 4/6 mid-systolic murmur was heard loudest over the left sternal border and observed to increase in intensity on Valsalva manoeuvre. Resting electrocardiography (ECG) showed sinus rhythm with left axis deviation. There was evidence of biatrial enlargement and left ventricular hypertrophy, based on voltage criteria. Ambulatory Holter monitoring did not reveal any episode of atrial fibrillation. Echocardiography showed severe diffuse asymmetrical septal hypertrophy measuring $2.64 \mathrm{~cm}$, and systolic anterior motion (SAM) (Figs. 1a \& b) with an LVOT gradient of $48 \mathrm{mmHg}$ at rest and $70 \mathrm{mmHg}$ during Valsalva manoeuvre (Fig. 2).

Optimisation of the patient's medical therapy was attempted by increasing his atenolol dose to $50 \mathrm{mg}$ bd.
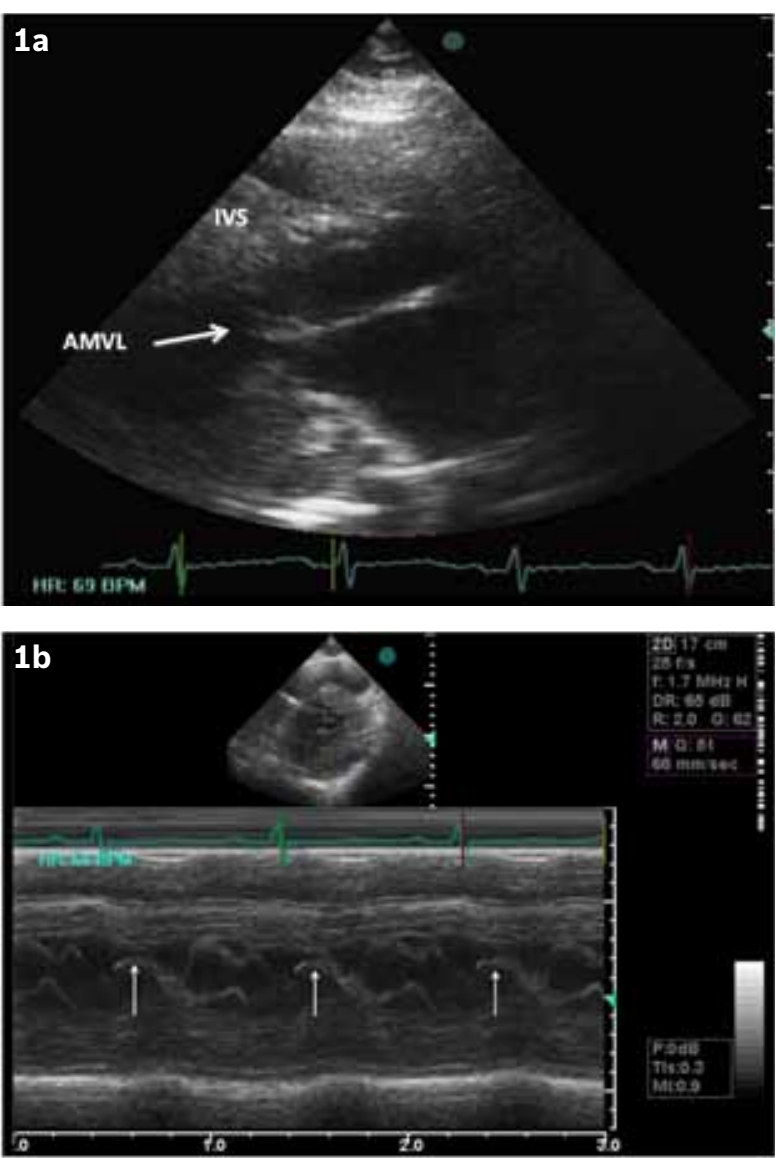

Fig. 1 (a) Echocardiogram shows the patient's heart. (b) M-mode echocardiogram shows systolic anterior motion (arrows) of the anterior mitral valve leaflet. AMVL: anterior mitral valve leaflet; IVS: interventricular septum; LVOT: left ventricular outflow tract

However, the patient promptly reported sexual dysfunction and increasing fatigue. The patient was given a trial of verapamil $240 \mathrm{mg}$ bd for one month, but his dyspnoea did not improve. He was then advised to undergo myectomy to relieve the LVOT gradient. However, after discussion of the relative risks and benefits of surgery, and the alternative

${ }^{1}$ Faculty of Medicine, The University of Melbourne, Melbourne, Australia, ${ }^{2}$ Parkway Heart and Vascular Centre, Mount Elizabeth Novena Specialist Centre, Singapore Correspondence: Mr Yee Sen Chan Wah Hak, Medical Student, Faculty of Medicine, Dentistry and Health Sciences Student Centre, Level 1, Brownless Biomedical Library, The University of Melbourne, Melbourne, Victoria 3010, Australia. yeesenchan@gmail.com 


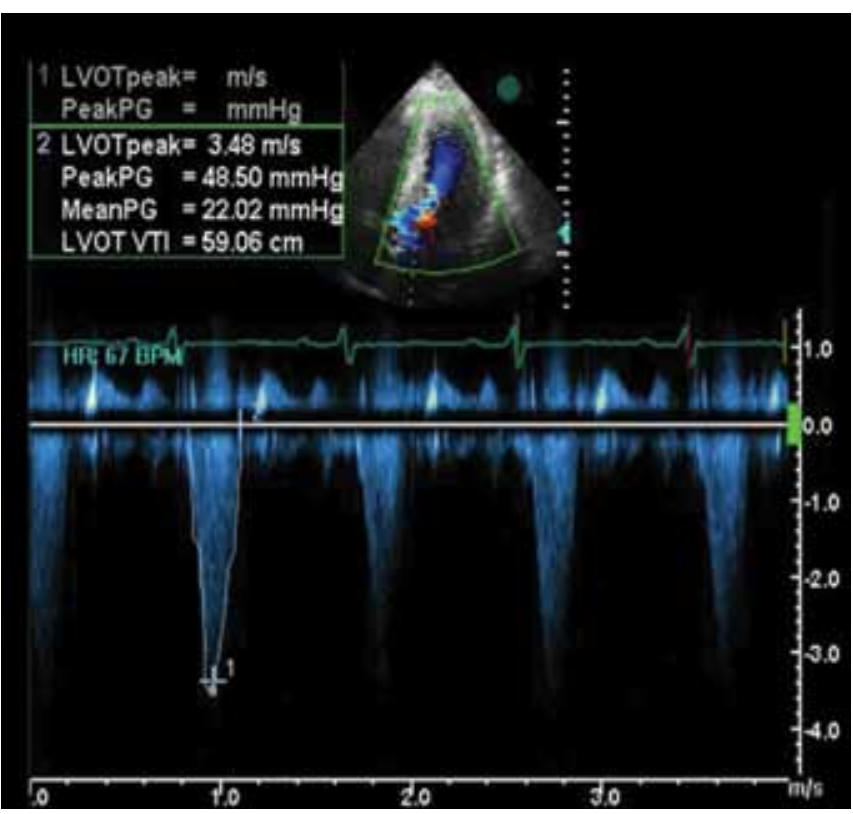

Fig. 2 Doppler recording shows left ventricular outflow tract gradient before septal artery embolisation.

options of alcohol septal ablation and coil embolisation, the patient chose to have nonsurgical septal reduction via coil embolisation, as he preferred a less invasive procedure.

Coronary angiography was performed via the right femoral approach following the insertion of a $6 \mathrm{~F}$ sheath, revealing the presence of normal coronary arteries with a large first septal branch in the left anterior descending artery. A bolus dose of 5,000 IU of heparin was given intravenously. A 6F EBU 3.75 guide catheter (Medtronic Cardiovascular, Santa Rosa, CA, USA) was used to cannulate the left coronary artery. A 0.014-inch Runthrough guidewire (Terumo Corporation, Tokyo, Japan) was advanced deep into the first septal branch, and a $2.7 \mathrm{~F}$ microcatheter (Progreat; Terumo Corporation, Tokyo, Japan) was slowly advanced into the septal artery over the guidewire. A VortX 18 diamond-shaped, fibred platinum coil (Boston Scientific, Watertown, NY, USA) was introduced in the microcatheter after the guidewire was removed. With a coil pusher, the coil was embolised to one of the branches of the first septal artery (S1[a]; Fig. 3a) so that the distal and proximal ends of the coil were inside the branch and the main septal artery, respectively. The 0.014 -inch Runthrough guidewire was then reintroduced into the microcatheter. Next, the second branch of the septal artery (S1[b]; Fig. 3a) was wired and the microcatheter was advanced into it. Likewise, a second, similar coil was embolised into the S1(b) branch of the first septal artery. Post embolisation, there was complete occlusion of the large, first septal artery and its two branches (Figs. 3a \& b).

The patient developed moderate chest pain after the procedure, requiring initial intravenous administration of a bolus dose of $5 \mathrm{mg}$ of morphine. The patient did not require any further analgesia. The patient was monitored on the catheter lab table for 30 mins and transferred to the coronary care unit (CCU) for further observation. His ECG in CCU showed
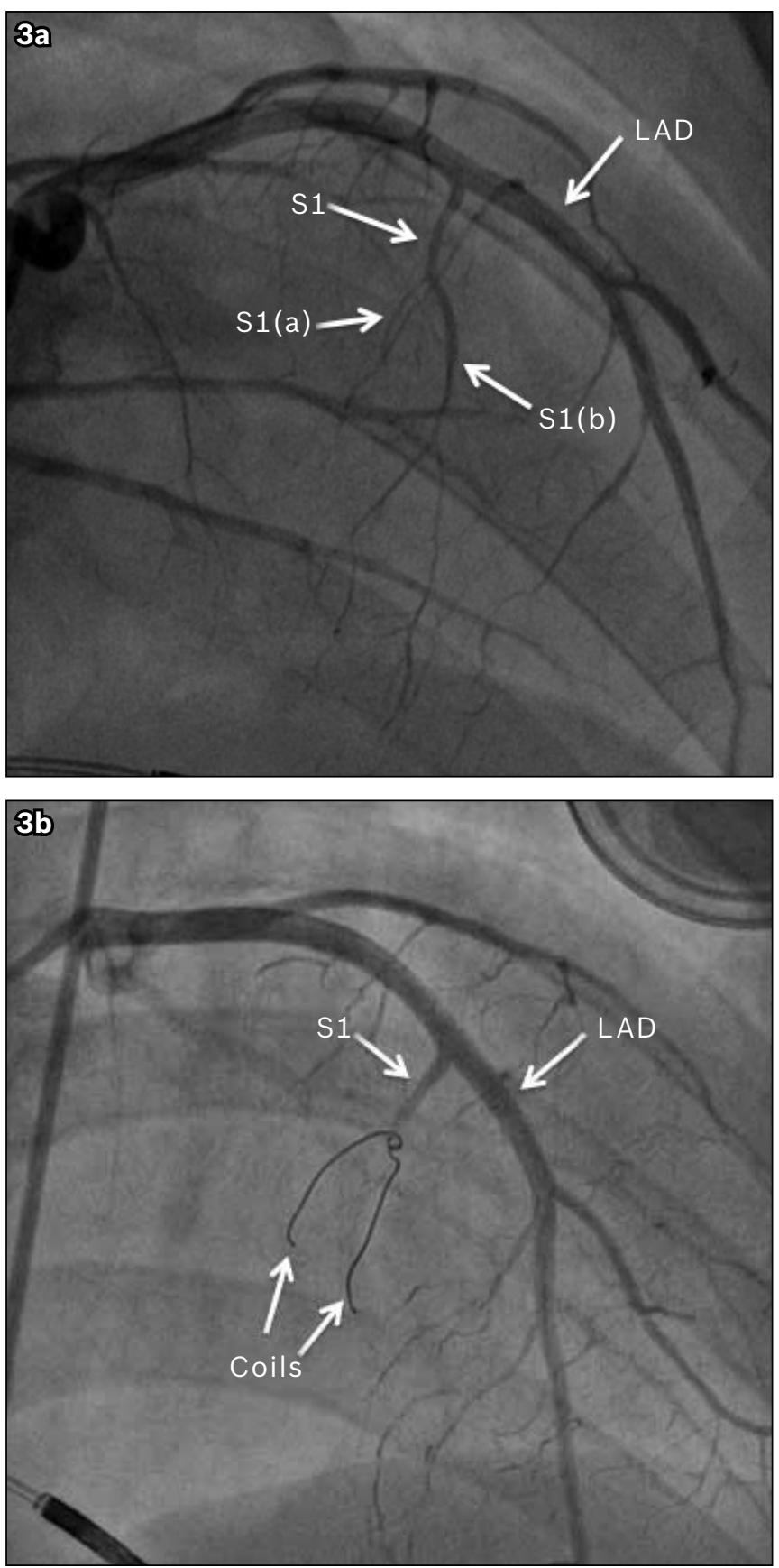

Fig. 3 (a) Left coronary angiogram at baseline shows the left anterior descending ( $L A D$ ) artery and the first septal artery (S1) with its two branches, denoted by $\mathrm{S} 1(\mathrm{a})$ and $\mathrm{S} 1$ (b). (b) Left coronary angiogram shows the position of the coil relative to the LAD and S1 after coil embolisation of the $\mathrm{S} 1$.

minimal ST elevation in lead $\mathrm{V} 3$ and $\mathrm{T}$ wave inversion in leads V5-V6. (Figs. 4a \& b). The next day, his creatine phosphokinase level peaked at $460 \mathrm{IU} / \mathrm{L}$ and his creatine kinase-MB level, at $16 \mathrm{mg} / \mathrm{mL}$. Echocardiography performed the following day showed hypokinesis of the basal septum and reduction of the patient's peak LVOT velocity to $38 \mathrm{mmHg}$ during Valsalva manoeuvre.

At follow-up 18 months later, the patient continued to do well with no reported symptoms of dyspnoea or presyncope. Repeat echocardiography showed a small reduction in septal thickness $(2.56 \mathrm{~cm})$, with a peak LVOT gradient of $24 \mathrm{mmHg}$ at rest and $36 \mathrm{mmHg}$ during Valsalva manoeuvre (Fig. 5). 

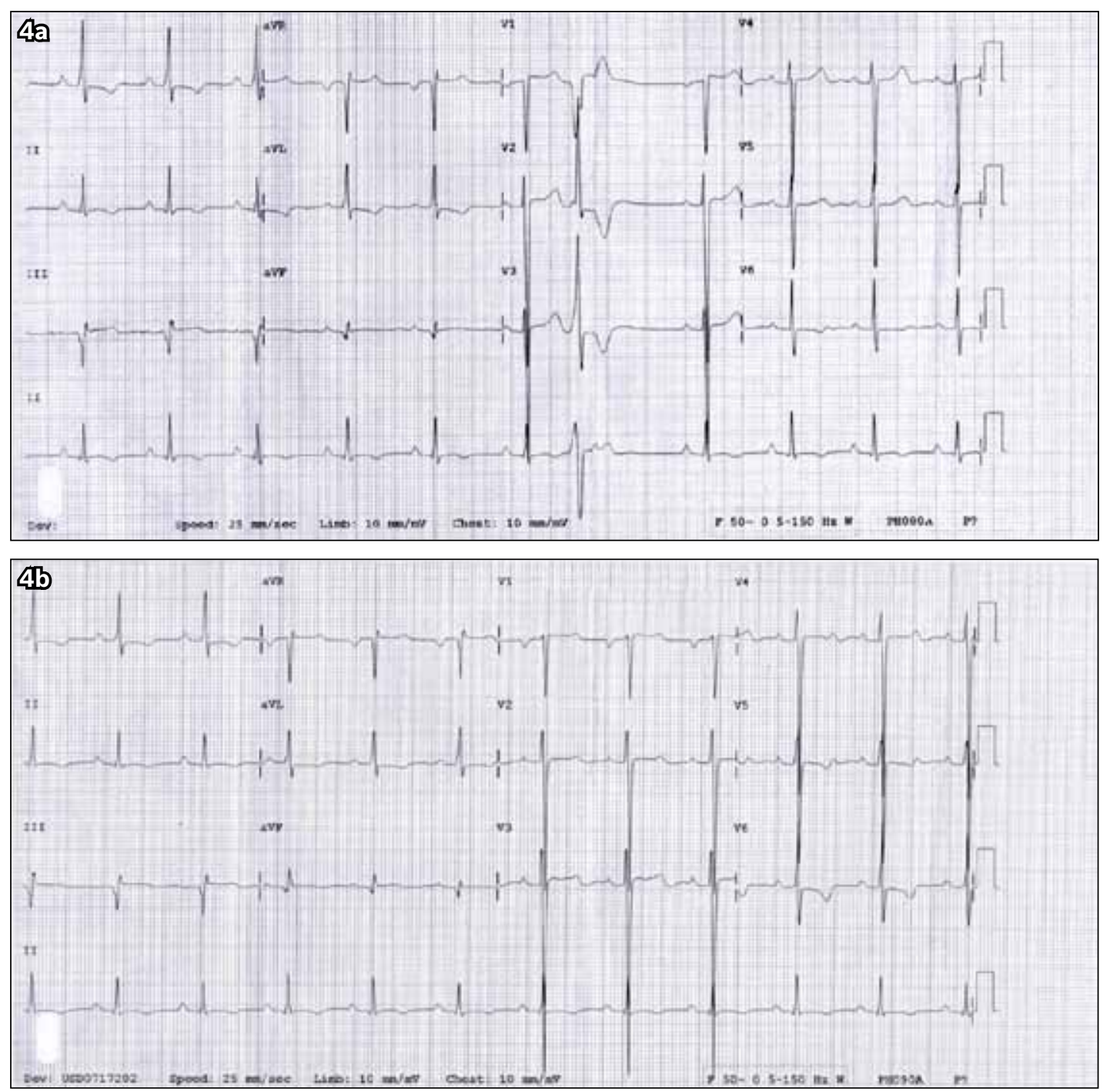

Fig. 4 ECGs show (a) sinus rhythm with occasional premature ventricular complex with $\mathrm{P}$ mitrale, increased precordial voltages with nonspecific ST-segment and T-wave abnormalities in leads I, AVL and V6, and deep narrow $Q$ wave in lead III before coil embolisation; and (b) minimal ST elevation in lead V3 and T-wave inversion in leads V5-V6 after coil emoblisation.

\section{DISCUSSION}

Surgical myectomy remains the gold standard for the treatment of refractory HOCM with significant outflow tract obstruction, especially in younger patients refractory to medications such as high dose beta-blockers, calcium blockers and disopyramide, or experiencing intolerable drug-related adverse effects. ${ }^{(1,2)}$ The operative mortality of surgical myectomy varies widely, but in large referral centres it can be as low as $1 \%-2 \%$. The surgical technique has evolved from the classic Morrow myotomy and myectomy, which uses two parallel longitudinal incisions in the septum, to a more extensive left ventricular septal myectomy toward the apex of the heart. In addition, all areas of papillary muscle fusion to the septum or ventricular free wall are divided. Anomalous chordal structures and fibrous attachment of the mitral valve leaflets to the ventricular septum are also excised. If myectomy is successful, there will be little or no residual LVOT gradient, and little or no SAM of the mitral valve. ${ }^{(3)}$

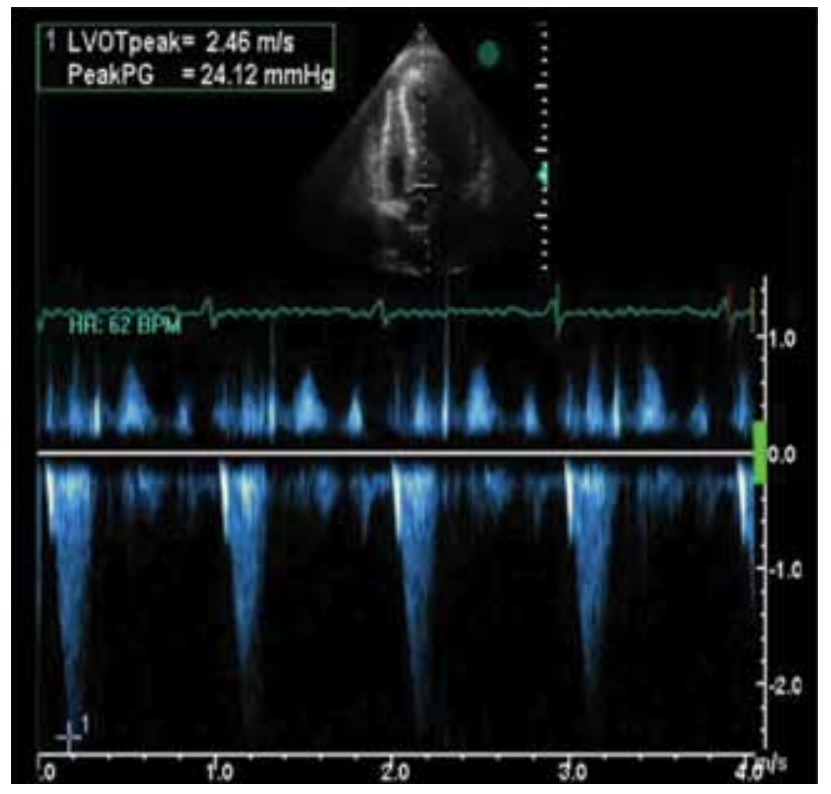

Fig. 5 Doppler recording at follow-up shows left ventricular outflow tract gradient post septal artery embolisation. 
More recently, alcohol septal ablation has emerged as an alternative to myectomy, and it has been shown to effectively reduce LVOT gradient by causing septal necrosis and scar formation. ${ }^{(3)}$ However, as ethanol penetrates more deeply into the capillary beds, an area of necrosis greater than anticipated can result from the procedure. Furthermore, alcohol has direct toxicity on the conduction tissue. Alcohol septal ablation has been shown to increase the risk of conduction abnormalities, which often require implantation of a permanent pacemaker, compared to surgical myectomy. ${ }^{(4,5)}$ Other serious complications include ventricular septal defect and lifethreatening ventricular arrhythmias due to the resulting scar tissue formation. The amount of ethanol injected and the number of septal arteries instilled with ethanol are major determinants of complications of alcohol septal ablation. ${ }^{(6)}$ Recently, in order to define the extent of myocardium that is at risk, so as to identify patients who are more likely to develop complications following the procedure, the usage of intraprocedural contrast echocardiography has been proposed.

Coil embolisation emerged as an alternative in our case, as our patient refused surgery and we wanted to avoid potential serious complications associated with alcohol ablation such as complete heart block and unintended spillage of alcohol into the left anterior descending artery, which could lead to severe complications. Coil embolisation technique is used in many settings, including the occlusion of small vessels such as intracranial aneurysms and patent ductus arteriosus, and during coronary interventions in cases of coronary perforation. The aim of performing coil embolisation in HOCM is to cause septal ischaemia and secondary necrosis. Durand et $\mathrm{al}^{(7)}$ described twenty patients with HOCM who had undergone septal reduction using coil embolisation; occlusion of the septal branches was successful in all patients, with no incidence of complete heart block or ventricular tachycardia at four months follow-up. However, one patient developed ventricular septal defect and died 19 days after the procedure. At six months follow-up, all the remaining patients reported improved symptoms, with echocardiography showing significant reduction in septal thickness $(21 \pm 3 \mathrm{~mm}$ vs. $17 \pm 4 \mathrm{~mm})$ and LVOT pressure gradient $(80 \pm 29 \mathrm{mmHg}$ vs. $35 \pm 29 \mathrm{mmHg}$. ${ }^{(7)}$ In our patient, repeat echocardiography performed one day after coil embolisation showed hypokinesis of the basal septum with a peak LVOT flow velocity of $2.5 \mathrm{~m} / \mathrm{s}$. Our patient continued to report symptomatic improvement 18 months after the procedure. Repeat echocardiography showed a small reduction in septal thickness and the patient's peak LVOT velocity was maintained at $36 \mathrm{mmHg}$ during Valsalva manoeuvre.

Coil embolisation is considered a palliative, and not curative, procedure. The other possible complications of coil embolisation include complete heart block and inadvertent delivery of the coil into the left anterior descending artery. This highlights the importance of choosing a good guide catheter with strong backup support, as was done in our case. The coils should also be delivered as distally as possible under continuous fluoroscopy. As this technique is relatively new, there is no current recommended guideline for this procedure. Furthermore, there is no direct comparison of coil embolisation with alcohol septal ablation. The major advantage of coil embolisation lies in its more predictable nature, and the avoidance of heart block and the widely reported unintentional alcohol spillage into the left anterior descending artery from alcohol ablation. We would typically recommend follow-up coronary angiography to ascertain that no contralateral collateral vessels have been formed. However, the patient has refused to undergo a repeat study thus far. To the best of our knowledge, this is the first local case of a patient with HOCM treated by nonsurgical septal reduction in Singapore.

\section{REFERENCES}

1. Maron B, McKenna WJ, Danielson GK, et al. American College of Cardiology/European Society of Cardiology Clinical Expert Consensus Document on Hypertrophic Cardiomyopathy. A report of the American College of Cardiology Foundation Task Force on Clinical Expert Consensus Documents and the European Society of Cardiology Committee for Practice Guidelines. Eur Heart J 2003; 24:1965-91.

2. Gersh BJ, Maron BJ, Bonow RO, et al. 2011 ACCF/AHA Guideline for the Diagnosis and Treatment of Hypertrophic Cardiomyopathy: a report of the American College of Cardiology Foundation/American Heart Association Task Force on Practice Guidelines. Developed in collaboration with the American Association for Thoracic Surgery, American Society of Echocardiography, American Society of Nuclear Cardiology, Heart Failure Society of America, Heart Rhythm Society, Society for Cardiovascular Angiography and Interventions, and Society of Thoracic Surgeons. J Am Coll Cardiol 2011; 58:e212-60.

3. Hess O, Sigwart U. New treatment strategies for hypertrophic obstructive cardiomyopathy: alcohol ablation of the septum: the new gold standard? J Am Coll Cardiol 2004; 44:2054-5.

4. Agarwal S, Tuzcu E, Desai MY, et al. Updated meta-analysis of septal alcohol ablation versus myectomy for hypertrophic cardiomyopathy. J Am Coll Cardiol 2010; 55:823-34

5. Talreja DR, Nishimura RA, Edwards WD, et al. Alcohol septal ablation versus surgical septal myectomy: comparison of effects on atrioventricular conduction tissue. J Am Coll Cardiol 2004; 44:2329-32.

6. Nagueh SF, Groves BM, Schwartz L, et al. Alcohol septal ablation for the treatment of hypertrophic obstructive cardiomyopathy. A multicenter North American registry. J Am Coll Cardiol 2011; 58:2322-8.

7. Durand E, Mousseaux E, Coste $\mathrm{P}$, et al. Non-surgical septal myocardial reduction by coil embolization for hypertrophic obstructive cardiomyopathy: early and 6 months follow-up. Eur Heart J 2008; 29:348-55. 